\title{
Plasma Dynamics During Breakdown in an HID Lamp
}

\author{
Ananth N. Bhoj and Mark J. Kushner, Fellow, IEEE
}

\begin{abstract}
Large starting voltages in metal halide lamps result in sputtering of the electrodes and reduced lamp life. A two-dimensional plasma hydrodynamics model was used to investigate breakdown in metal halide lamp. Images depicting the progress of the breakdown in Ar during cold startup are presented.
\end{abstract}

Index Terms-Avalanche, breakdown, HID, lamp, modeling, plasma.

$\mathbf{M}$ ETAL halide arc lamps are widely used sources of indoor and large area outdoor illumination due to their favorable color characteristics and high efficiency [1]. The cold fill of such lamps contains 10s of torr of a rare gas, typically Ar, with the vapor pressure of condensed metal and metal-halide salts. The electrical breakdown during startup produces warming of the discharge tube and vaporization of the metal (-halide) salts, resulting in a multiatmosphere plasma at steady state. The high voltages needed to breakdown the gas may sputter electrode material and decrease the life of the lamp. In order to facilitate breakdown of the cold gas, techniques such as auxiliary electrodes and ultraviolet (UV) radiation sources are often employed to create seed electrons in the discharge tube [2].

A two-dimensional plasma hydrodynamics model [3] was implemented on a geometry closely resembling a commercial polycrystalline alumina metal halide lamp to simulate breakdown processes during cold startup. The variables solved are the electric potential from Poisson's equation and species densities from multifluid transport equations. Gain and loss terms include sources from electron impact ionization and excitation; and electron secondary emission and photoionization due to external UV sources and internally generated photons. Sources due to secondary electrons emitted from the cathode are accounted for using a Monte Carlo simulation. The species included in the simulation are $\mathrm{Ar}, \mathrm{Ar}^{*}, \mathrm{Ar}^{+}, \mathrm{Ar}^{* *}, \mathrm{Ar}_{2}^{*}$, and $\mathrm{Ar}_{2}^{+}$. The model uses unstructured meshes created using SkyMesh2 [4], a commercial mesh generator. Individual images were created from the raw data from the model using Tecplot v8 [5] and Corel Draw v12 [6], and assembled into the final image using Adode Photoshop v7 [7].

The cylindrically symmetric discharge tube is $1 \mathrm{~cm}$ in diameter. The upper electrode is the powered anode and the lower

Manuscript received July 8, 2004; revised November 7, 2004. Work supported in part by National Science Foundation CTS03-15353 and in part by the General Electric Research and Development Center.

A. N. Bhoj is with the Department of Chemical and Biomolecular Engineering, University of Illinois, Urbana, IL 61801 USA (e-mail: bhoj@uiuc.edu).

M. J. Kushner is with the Department of Electrical and Computer Engineering, Iowa State University, Ames, IA, 50011 USA (e-mail: mjk@iastate.edu).

Digital Object Identifier 10.1109/TPS.2005.845931 electrode is the grounded cathode. The interelectrode gap is $1.6 \mathrm{~cm}$. The voltage applied to the anode is $2000 \mathrm{~V}$ with a rise time of $150 \mathrm{~ns}$.

The progress of the breakdown is shown in Fig. 1 for Ar at 30 torr by images of the electron density and electron impact source functions at various times. Electric field vectors (relative scaling) are superimposed on the source functions. An enlargement of the avalanche head at $175 \mathrm{~ns}$ is also shown. At $125 \mathrm{~ns}$, the electric field in the vicinity of the anode rises and is geometrically enhanced due to the electrode structure. Peak electric fields of 700-800 Td $\left(1 \mathrm{Td}=10^{-17} \mathrm{~V}-\mathrm{cm}^{2}\right)$ elevate the electron temperature $\mathrm{T}_{\mathrm{e}}$, producing large electron impact sources close to the anode, and the electron density begins to avalanche. By $175 \mathrm{~ns}$, the increasingly conductive plasma expels the electric field. The peak electric field then moves toward the cathode, aided by photoionization.

A cathode directed streamer-like structure develops having a large electric field due to space charge separation at its head. $\mathrm{T}_{\mathrm{e}}$ increases in the in the avalanche front producing large electron impact sources which also traverse the gap. The electron density rises in the ionized region behind the avalanche front, compressing the applied voltage into the unionized region ahead of the avalanche front. Although electron sources are largest at the head (lower tip) of the avalanche, nonnegligible sources also occur on its radial boundary where electric fields are large due to charge separation. At $275 \mathrm{~ns}$, the avalanche is past halfway down the tube and the electric field vectors begin turning inwards toward the cathode. Their magnitudes increase due to both geometrical enhancement and by compression of the applied voltage that has been expelled from the high conductivity regions behind the avalanche front. Secondary electron emission from the cathode significantly contributes to ionization in these large electric fields by $300 \mathrm{~ns}$. At gap closure, an anode directed avalanche front is initiated.

\section{REFERENCES}

[1] S. T. Henderson and A. M. Marsden, Eds., Lamps and Lighting. New York: Crane, Russack and Co, Inc., 1972.

[2] W. W. Byszewski, Y. M. Li, A. B. Budinger, and P. D. Gregor, "Advances in starting high-intensity discharge lamps," Plasma Sources Sci. Technol., vol. 5, pp. 720-735, 1996.

[3] M. J. Kushner, "Modeling of microdischarge devices: Pyramidal structures," J. Appl. Phys., vol. 95, pp. 846-859, 2004.

[4] Skyblue Systems Inc., Troy, NY.. [Online]. Available: http://www.skybluesystems.com

[5] Tecplot Inc., Bellevue, WA.. [Online]. Available: http://www.tecplot.com

[6] Corel Corp., Ottawa, ON, Canada.. [Online]. Available: http://www.corel.com

[7] Adobe Corp., San Jose, CA, Canada.. [Online]. Available: http://www.adobe.com 

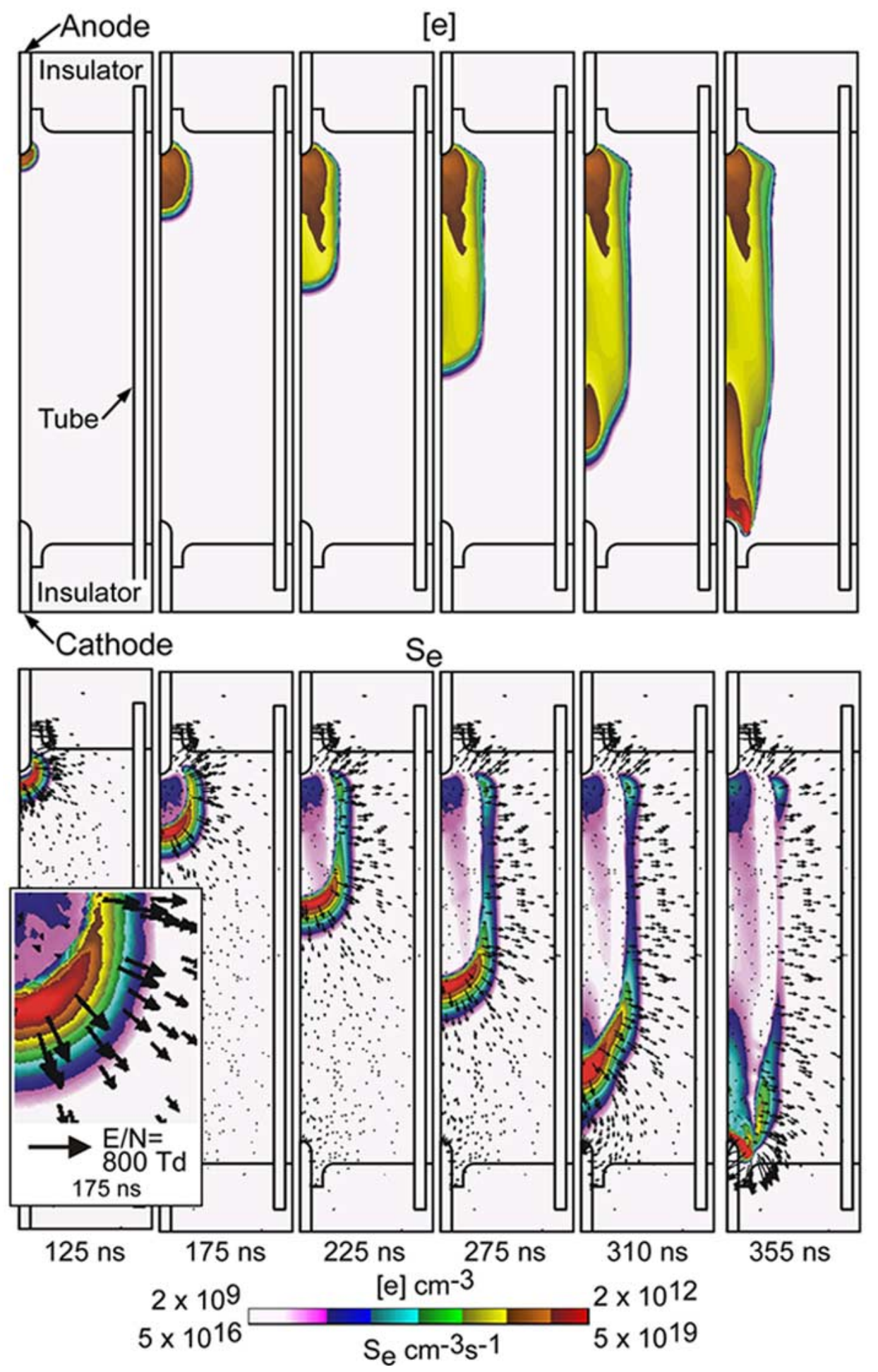

Fig. 1. Plasma characteristics during breakdown in Ar at 30 torr in a metal halide lamp. (Top) Electron density. (Bottom) Electron impact ionization sources with superimposed electric field vectors. Vector symbols have relative scaling with respect to the magnitude of the field. An enlargement of the avalanche head at $175 \mathrm{~ns}$ is shown by the inset. 